\title{
A Review on Reversible Logic Gates and it's QCA Implementation
}

\author{
Mohammad Abdullah-Al- \\ Shafi \\ Dept of ICT \\ Mawlana Bhashani Science \\ and Technology University \\ Tangail, Bangladesh
}

\author{
Md Shifatul Islam \\ Dept of ICT \\ Mawlana Bhashani Science \\ and Technology University \\ Tangail, Bangladesh
}

\author{
Ali Newaz Bahar \\ Dept of ICT \\ Mawlana Bhashani Science \\ and Technology University \\ Tangail, Bangladesh
}

\begin{abstract}
Quantum Dot Cellular Automata (QCA) is a rising innovation which seems to be a good competitor for the next generation of digital systems and widely utilized as a part of advanced frameworks. It is an appealing substitute to ordinary CMOS innovation because of diminutive size, faster speed, extremely scalable feature, ultralow power consumption and better switching frequency. The realization of quantum computation is not possible without reversible logic. Reversible logic has enlarged operations in quantum computation. Generally reversible computing is executed when system composes of reversible gates. It has numerous fields of use as applied science, quantum dot cellular automata as well as low power VLSI circuits, low power CMOS, digital signal processing, computer graphics. In this paper, the quantum implementation of primitive reversible gate has been presented. The proposed gates have been designed and simulated using QCADesigner.
\end{abstract}

\section{General Terms}

Quantum Cellular Automata and Reversible Logic Gates

\section{Keywords}

Quantum-dot Cellular Automata (QCA), Reversible logic, Reversible gates, QCA Designer

\section{INTRODUCTION}

Nanotechnology allows new measurements for computing as typical silicon transistor technology faces disparate complication due to its speed, power utilization and challenges in feature size diminishment. Among promising technologies, Quantum dot Cellular Automata (QCA) [1, 2] assurance aforementioned components. Quantum Dot Cellular Automata (QCA) has been utilized widely and wipes out these issues. Quantum Dot Cellular Automata (QCA) is a productive substitute of Complementary metal oxide semiconductor (CMOS) innovation [3]. QCA is a combined strategy for transmission and computation $[4,5,6]$. The reversible logic circuits design the key structural engineering of quantum computers as all quantum processes are reversible. Efficiency loss is a vital consideration in planning digital framework. Energy loss because of data fall in circuits and systems constructed using irreversible logic circuit was exhibited by R. Landauer. From Landauer's principle, the loss of one bit of information lost, will exhaust $k T \ln (2)$ joules of vitality where $k=1.38 \times 10^{-23} \mathrm{JK}^{-1}$ is the Boltzmann's constant and $\mathrm{T}$ is supreme temperature in Kelvin [7]. Bennett demonstrate that to sidestep $k \mathrm{~T} \ln 2$ joules of vitality dissipation in a circuit, it must be manufactured from reversible circuits [8]. In reversible logic circuits information fall is not feasible so it is preferable to form combinational circuit. Reversible logic circuits are of high enthusiasm for nano innovation [9], quantum computing [10] and optical computing [11]. An exceptional utilization of reversible logic circuit lies in quantum computers [12]. Number of input and output is identical in reversible logic gate to have one to one mapping. Classical logic combination procedures cannot be directly connected to model reversible circuit. An imperative parameter of a reversible logic circuit is garbage output. Generally an unutilized product from a gate is garbage. The quantum cost is a cost that is associated with every reversible logic gate. In this paper, mostly available reversible logic gates and their QCA design are presented.

\section{BASIC TERMS RELATED TO REVERSIBLE LOGIC}

A. Reversible Function: The Boolean function $\mathrm{F}(\mathrm{x} 1 ; \mathrm{x} 2, \ldots \mathrm{xn})$ with multiple output of $n$ Boolean variables is called reversible if the number of output is identical to number of inputs. Any output pattern has a unique pre-image. Particularly, reversible functions are those that execute permutations of the set of input vectors [13].

B. Reversible logic: In reversible logic, the numbers of input are equal to number the outputs of the gates. This generates particular set of output vector for each set of input vector.

C. Garbage outputs: Additional inputs or outputs can be added so as to make the number of inputs and outputs identical whenever required. The number of outputs added to make an m-input-k-output function $((\mathrm{m} ; \mathrm{k})$ function) reversible is called garbage. The formula between the number of garbage outputs and constant inputs are shown below -

Input + constant input $=$ output + garbage

An output line that is necessary to sustain the reversibility is referred as garbage line [14].

D. Quantum Cost: Quantum cost refers the amount of effort needed to transform a reversible circuit to a quantum circuit. It is calculated knowing the number of primitive reversible logic gates needed to recognize the circuit.

E. Ancilla inputs: Ancilla inputs or constant inputs is the number of inputs which are maintain constant either 0 or 1 in order to incorporate the given logical function [15].

F. Flexibility: Flexibility is the universality of a reversible logic gate for realizing more functions [16].

G. Gate level: In the circuit gate level is the number of levels that are needed to recognize the given logic functions.

H. Hardware complexity: Hardware Complexity is the overall number of logic action in a circuit. In other words the total number of AND, OR and EXOR operation in a circuit [17, $18]$. 


\section{QCA BASIC}

The primary architecture block of QCA is QCA cell. It can be viewed as an arrangement of four dots located at corners of a square [19]. Depending on the electron's location, QCA cell has two form of polarization, binary 1 or $P=+1.00$ and binary 0 or $P=-1.00$ [17] shown in figure 1. QCA offer an innovative distinct option for the transistor paradigm [20, 21]. The cell polarization defined as follows:

$P=\frac{\left(\rho_{2}+\rho_{4}\right)-\left(\rho_{1}+\rho_{3}\right)}{\left(\rho_{1}+\rho_{2}+\rho_{3}+\rho_{4}\right)}$

Where, the electronic charge denoted as $\rho_{i}$

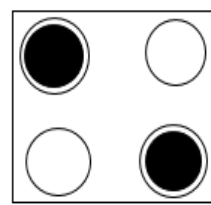

$\mathrm{P}=-1.00$

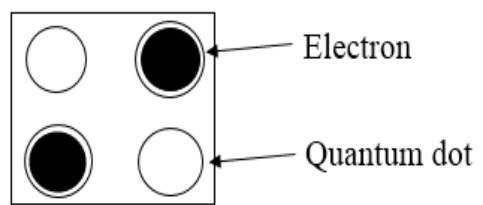

$\mathrm{P}=+1.00$
Fig 1: Four doted QCA cell

\subsection{QCA Wire}

QCA wire is a combination of interconnected QCA cells with similar polarization. It used to carry signal from one direction to another. Therefore QCA wires can propagate data from one side to another [22].

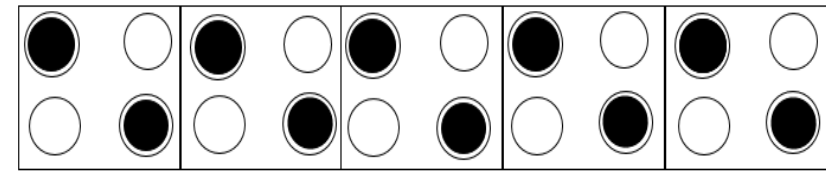

Fig 2: QCA wire

\subsection{QCA Inverter}

QCA inverter returns the reversed value of the input one and it consists of four QCA wire shown in figure 3.

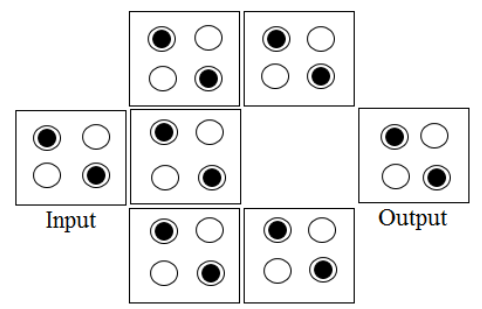

Fig 3: QCA inverter

\subsection{QCA Majority Gate}

Majority gate or voter [23] (MV) is described as logic function $\mathrm{MV}(\mathrm{A}, \mathrm{B}, \mathrm{C})=\mathrm{AB}+\mathrm{AC}+\mathrm{BC}$. Majority gate can be used as AND gate and OR gate. For AND gate the input of the voter is zero and for OR gate the input is 1 .

$\operatorname{MV}(\mathrm{A}, \mathrm{B}, 0)=\mathrm{AB}+\mathrm{A} \cdot 0+\mathrm{B} \cdot 0=\mathrm{AB}$

$\operatorname{MV}(\mathrm{A}, \mathrm{B}, 1)=\mathrm{AB}+\mathrm{A} \cdot 1+\mathrm{B} \cdot 1=\mathrm{A}+\mathrm{B}$

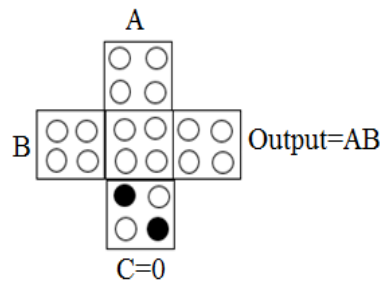

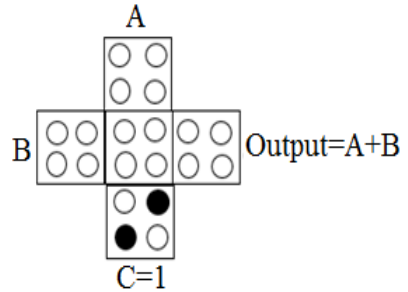

Fig 4: Majority voter as 2-input AND gate and 2-input OR gate

\section{BASIC REVERSIBLE GATES}

A gate is reversible if there is a one to one correspondence between its input and output assignments. Reversible gate realizes one to one logic, thus practically it is a many input output gate [24]. Many reversible gates exist already. A reversible circuit should be formed using minimal number of reversible gates. To determine the complexity and performance of reversible gate some specifications must be viewed. These are the number of reversible gates, number of ancilla inputs, number of garbage outputs and quantum cost. Some of the essential reversible gates are:

\subsection{NOT Gate}

NOT gate is a $1^{*} 1$ gate and an elementary gate. The quantum cost of not gate is zero.

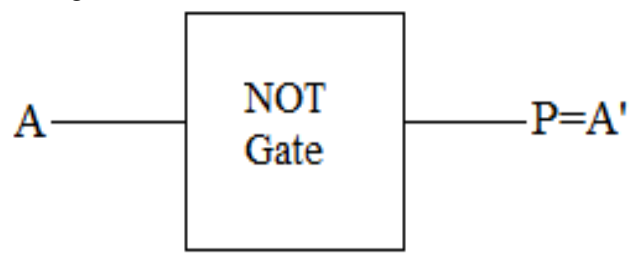

Fig 5: NOT gate

Table 1. Truth table of NOT gate

\begin{tabular}{|c|c|}
\hline Input & Output \\
\hline $\mathrm{A}$ & $\mathrm{A}^{\prime}$ \\
\hline 1 & 0 \\
\hline 0 & 1 \\
\hline
\end{tabular}

\subsection{CNOT Gate}

It is a $2 * 2$ reversible gate also known as controlled not gate. Quantum cost of CNOT gate is one.

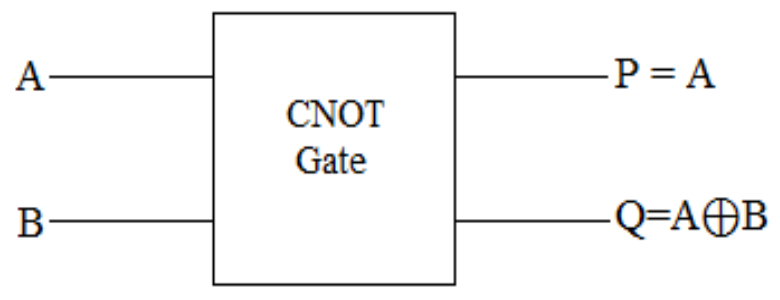

Fig 6: CNOT gate

Table 2. Truth table of CNOT gate

\begin{tabular}{|c|c|c|c|}
\hline \multicolumn{2}{|c|}{ Input } & \multicolumn{2}{c|}{ Output } \\
\hline $\mathrm{A}$ & $\mathrm{B}$ & $\mathrm{P}$ & $\mathrm{Q}$ \\
\hline 0 & 0 & 0 & 0 \\
\hline 0 & 1 & 0 & 1 \\
\hline 1 & 0 & 1 & 1 \\
\hline 1 & 1 & 1 & 0 \\
\hline
\end{tabular}




\subsection{FEYNMAN Gate}

A Feynman [25] gate is a $2 * 2$ gate and also called as controlled NOT. It is widely used for fan-out purposes. It has quantum cost one.

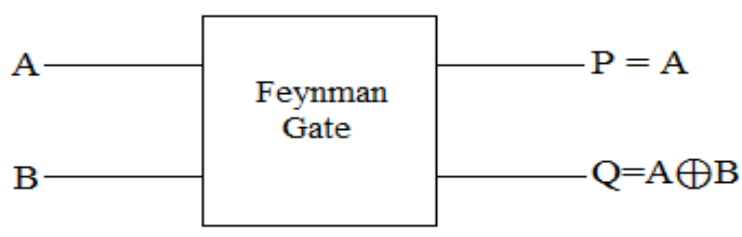

Fig 7: Feynman gate

Table 3. Truth table of Feynman gate

\begin{tabular}{|c|c|c|c|}
\hline \multicolumn{2}{|c|}{ Input } & \multicolumn{2}{c|}{ Output } \\
\hline $\mathrm{A}$ & $\mathrm{B}$ & $\mathrm{P}$ & $\mathrm{Q}$ \\
\hline 0 & 0 & 0 & 0 \\
\hline 0 & 1 & 0 & 1 \\
\hline 1 & 0 & 1 & 1 \\
\hline 1 & 1 & 1 & 0 \\
\hline
\end{tabular}

\subsection{Double Feynman Gate (F2G)}

Double Feynman $[26,27]$ gate is a $3 * 3$ gate. The outputs are defined by $\mathrm{P}=\mathrm{A}, \mathrm{Q}=\mathrm{A} \oplus \mathrm{B}, \mathrm{R}=\mathrm{A} \oplus \mathrm{C}$. It has quantum cost two.

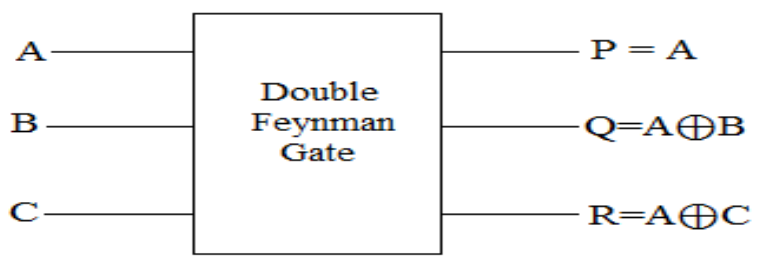

Fig 8: Double Feynman gate

Table 4. Truth table of Double Feynman gate

\begin{tabular}{|c|c|c|c|c|c|}
\hline \multicolumn{3}{|c|}{ Input } & \multicolumn{3}{|c|}{ Output } \\
\hline $\mathrm{A}$ & $\mathrm{B}$ & $\mathrm{C}$ & $\mathrm{P}$ & $\mathrm{Q}$ & $\mathrm{R}$ \\
\hline 0 & 0 & 0 & 0 & 0 & 0 \\
\hline 0 & 0 & 1 & 0 & 0 & 1 \\
\hline 0 & 1 & 0 & 0 & 1 & 0 \\
\hline 0 & 1 & 1 & 0 & 1 & 1 \\
\hline 1 & 0 & 0 & 1 & 1 & 1 \\
\hline 1 & 0 & 1 & 1 & 1 & 0 \\
\hline 1 & 1 & 0 & 1 & 0 & 1 \\
\hline 1 & 1 & 1 & 1 & 0 & 0 \\
\hline
\end{tabular}

\subsection{TOFFOLI Gate}

Toffoli gate $[28,29]$ is a $3 * 3$ reversible gate. The outputs are $\mathrm{P}=\mathrm{A}, \mathrm{Q}=\mathrm{B}, \mathrm{R}=\mathrm{AB} \oplus \mathrm{C}$. The quantum cost is five.

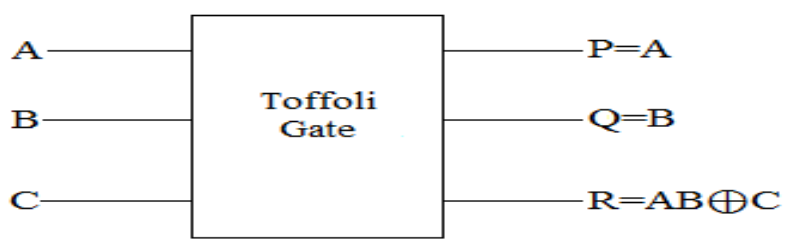

Fig 9: Toffoli gate
Table 5. Truth table of Toffoli gate

\begin{tabular}{|c|c|c|c|c|c|}
\hline \multicolumn{3}{|c|}{ Input } & \multicolumn{3}{c|}{ Output } \\
\hline A & B & C & P & Q & R \\
\hline 0 & 0 & 0 & 0 & 0 & 0 \\
\hline 0 & 0 & 1 & 0 & 0 & 1 \\
\hline 0 & 1 & 0 & 0 & 1 & 0 \\
\hline 0 & 1 & 1 & 0 & 1 & 1 \\
\hline 1 & 0 & 0 & 1 & 0 & 0 \\
\hline 1 & 0 & 1 & 1 & 0 & 1 \\
\hline 1 & 1 & 0 & 1 & 1 & 1 \\
\hline 1 & 1 & 1 & 1 & 1 & 0 \\
\hline
\end{tabular}

\subsection{FREDKIN Gate}

Fredkin $[30,31]$ gate which is a $3 * 3$ gate. The outputs of Fredkin gate are $\mathrm{P}=\mathrm{A}, \mathrm{Q}=\mathrm{A}^{\prime} \mathrm{B}+\mathrm{AC}, \mathrm{R}=\mathrm{AB}+\mathrm{A}^{\prime} \mathrm{C}$. It has quantum cost five.

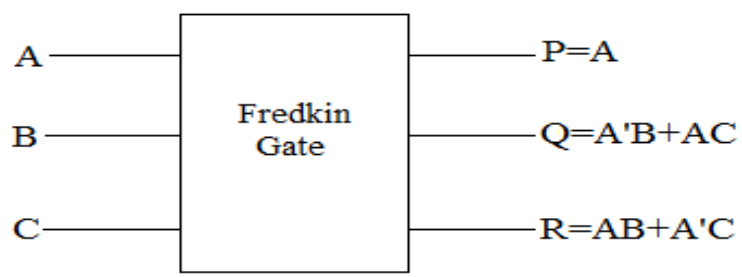

Fig 10: Fredkin gate

Table 6. Truth table of Fredkin gate

\begin{tabular}{|c|c|c|c|c|c|}
\hline \multicolumn{3}{|c|}{ Input } & \multicolumn{3}{c|}{ Output } \\
\hline A & B & C & P & Q & R \\
\hline 0 & 0 & 0 & 0 & 0 & 0 \\
\hline 0 & 0 & 1 & 0 & 0 & 1 \\
\hline 0 & 1 & 0 & 0 & 1 & 0 \\
\hline 0 & 1 & 1 & 0 & 1 & 1 \\
\hline 1 & 0 & 0 & 1 & 0 & 0 \\
\hline 1 & 0 & 1 & 1 & 1 & 0 \\
\hline 1 & 1 & 0 & 1 & 0 & 1 \\
\hline 1 & 1 & 1 & 1 & 1 & 1 \\
\hline
\end{tabular}

\subsection{PERES Gate}

Peres $[32,33]$ gate is a $3 * 3$ gate. The outputs $\mathrm{P}=\mathrm{A}, \mathrm{Q}=$ $\mathrm{A} \oplus \mathrm{B}, \mathrm{R}=\mathrm{AB} \oplus \mathrm{C}$. It has Quantum cost four. It has the minimum quantum cost, among $3 \times 3$ reversible gates.

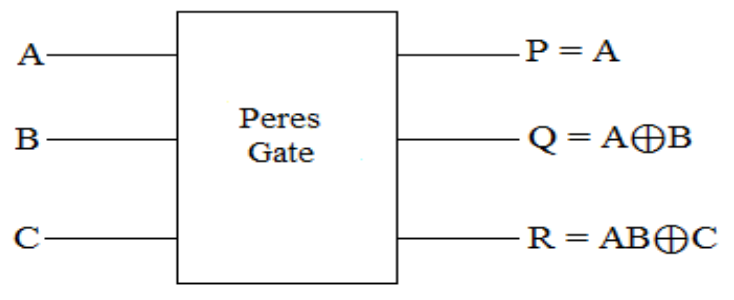

Fig 11: Peres gate 
Table 7. Truth table of Peres gate

\begin{tabular}{|c|c|c|c|c|c|}
\hline \multicolumn{3}{|c|}{ Input } & \multicolumn{3}{c|}{ Output } \\
\hline A & B & C & P & Q & R \\
\hline 0 & 0 & 0 & 0 & 0 & 0 \\
\hline 0 & 0 & 1 & 0 & 0 & 1 \\
\hline 0 & 1 & 0 & 0 & 1 & 0 \\
\hline 0 & 1 & 1 & 0 & 1 & 1 \\
\hline 1 & 0 & 0 & 1 & 1 & 0 \\
\hline 1 & 0 & 1 & 1 & 1 & 1 \\
\hline 1 & 1 & 0 & 1 & 0 & 1 \\
\hline 1 & 1 & 1 & 1 & 0 & 0 \\
\hline
\end{tabular}

\subsection{R Gate}

$\mathrm{R}[34,35]$ gate is a $3 \times 3$ gate . The outputs are $\mathrm{P}=\mathrm{A} \oplus \mathrm{B}, \mathrm{Q}$ $=\mathrm{A}, \mathrm{R}=\mathrm{C}^{\prime} \oplus(\mathrm{A} . \mathrm{B})$

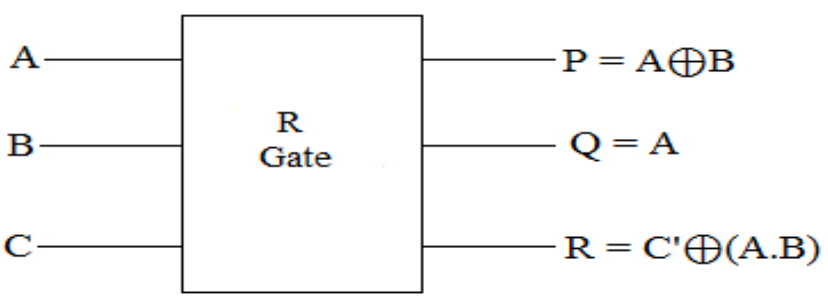

Fig 12: R gate

Table 8. Truth table of $R$ gate

\begin{tabular}{|c|c|c|c|c|c|}
\hline \multicolumn{3}{|c|}{ Input } & \multicolumn{3}{|c|}{ Output } \\
\hline $\mathrm{A}$ & $\mathrm{B}$ & $\mathrm{C}$ & $\mathrm{P}$ & $\mathrm{Q}$ & $\mathrm{R}$ \\
\hline 0 & 0 & 0 & 0 & 0 & 1 \\
\hline 0 & 0 & 1 & 0 & 0 & 0 \\
\hline 0 & 1 & 0 & 1 & 0 & 1 \\
\hline 0 & 1 & 1 & 1 & 0 & 0 \\
\hline 1 & 0 & 0 & 1 & 1 & 1 \\
\hline 1 & 0 & 1 & 1 & 1 & 0 \\
\hline 1 & 1 & 0 & 0 & 1 & 0 \\
\hline 1 & 1 & 1 & 0 & 1 & 1 \\
\hline
\end{tabular}

\subsection{BJN Gate}

$\mathrm{BJN}$ [25] gate is a $3 \times 3$ gate. The outputs are $\mathrm{P}=\mathrm{A}, \mathrm{Q}=\mathrm{B}, \mathrm{R}$ $=(\mathrm{A}+\mathrm{B}) \oplus \mathrm{C}$. The quantum cost of BJN Gate is five.

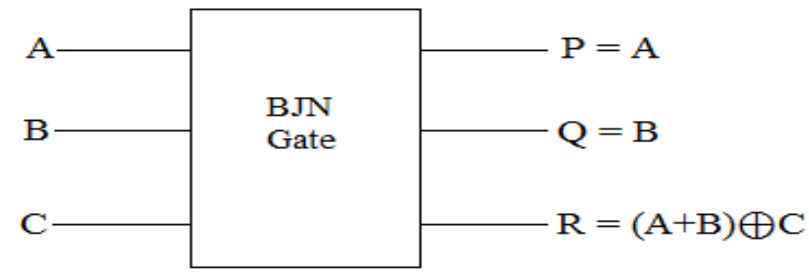

Fig 13: BJN gate
Table 9. Truth table of BJN gate

\begin{tabular}{|c|c|c|c|c|c|}
\hline \multicolumn{3}{|c|}{ Input } & \multicolumn{3}{c|}{ Output } \\
\hline A & B & C & P & Q & R \\
\hline 0 & 0 & 0 & 0 & 0 & 0 \\
\hline 0 & 0 & 1 & 0 & 0 & 1 \\
\hline 0 & 1 & 0 & 0 & 1 & 1 \\
\hline 0 & 1 & 1 & 0 & 1 & 0 \\
\hline 1 & 0 & 0 & 1 & 0 & 1 \\
\hline 1 & 0 & 1 & 1 & 0 & 0 \\
\hline 1 & 1 & 0 & 1 & 1 & 1 \\
\hline 1 & 1 & 1 & 1 & 1 & 0 \\
\hline
\end{tabular}

\subsection{MCL gate}

MCL [36] gate is a $3 \times 3$ gate. The outputs are $P=B^{\prime} C^{\prime}, Q=$ $\mathrm{A}^{\prime} \mathrm{B}^{\prime}, \mathrm{R}=\mathrm{A}$.

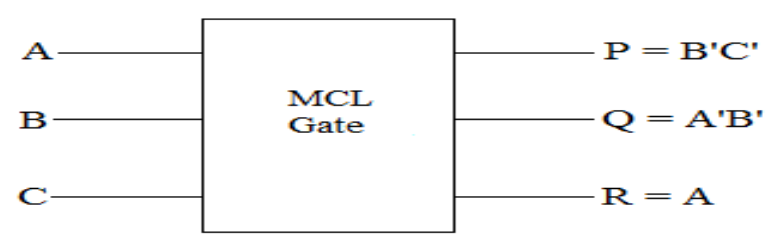

Fig 14: MCL gate

Table 10. Truth table of MCL gate

\begin{tabular}{|c|c|c|c|c|c|}
\hline \multicolumn{3}{|c|}{ Input } & \multicolumn{3}{c|}{ Output } \\
\hline $\mathrm{A}$ & $\mathrm{B}$ & $\mathrm{C}$ & $\mathrm{P}$ & $\mathrm{Q}$ & $\mathrm{R}$ \\
\hline 0 & 0 & 0 & 1 & 1 & 0 \\
\hline 0 & 0 & 1 & 0 & 1 & 0 \\
\hline 0 & 1 & 0 & 0 & 0 & 0 \\
\hline 0 & 1 & 1 & 0 & 0 & 0 \\
\hline 1 & 0 & 0 & 1 & 0 & 1 \\
\hline 1 & 0 & 1 & 0 & 0 & 1 \\
\hline 1 & 1 & 0 & 0 & 0 & 1 \\
\hline 1 & 1 & 1 & 0 & 0 & 1 \\
\hline
\end{tabular}

\section{QCA IMPLEMENTATION OF REVERSIBLE LOGIC GATES}

The design level is tested several approximate simulators such as the nonlinear approximation approaches and bistable simulation. But these schemes do not produce the actual results. Finally QCA Designer 2.0.3 is selected and this simulation engine is described [37]. QCA Designer is a tool used for design and simulation of QCA based circuits. The accuracy of these circuits is tested by the simulation tools of QCA Designer 2.0.3 [38]. The technical implementation is shown below.

$\mathrm{A}^{\prime}$

000000 000000

Fig 15: Simulated design of NOT gate 


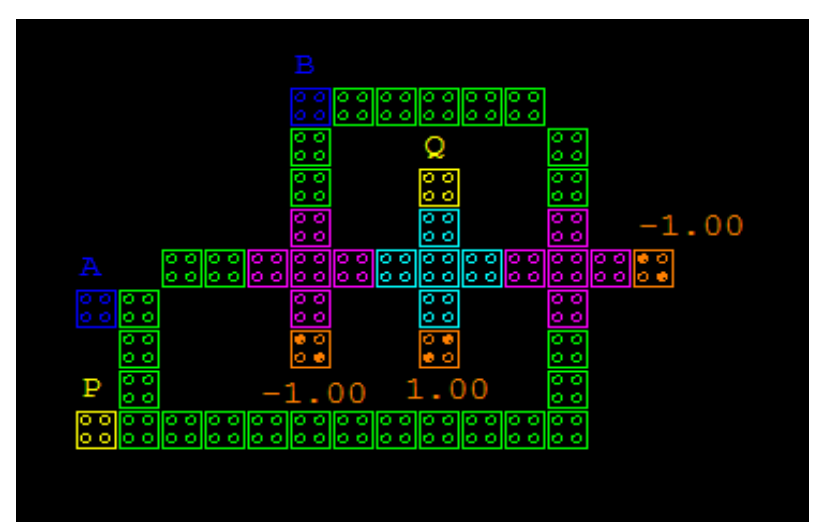

Fig 16: Simulated design of CNOT gate

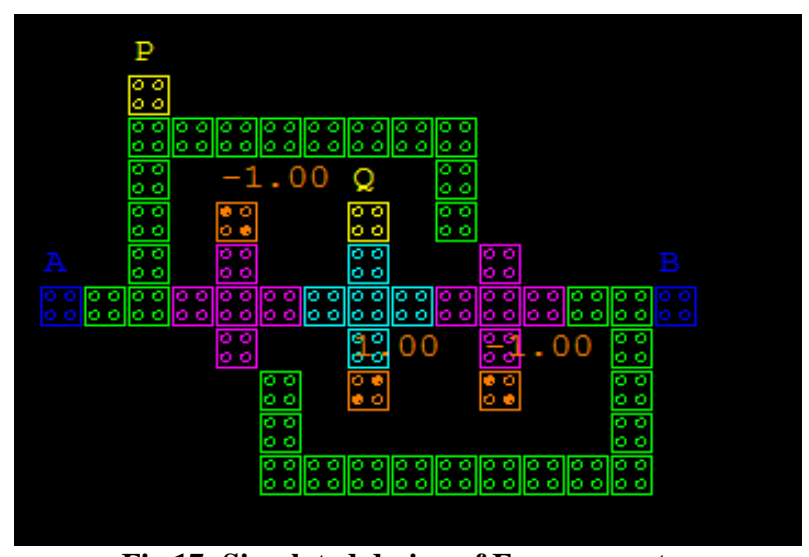

Fig 17: Simulated design of Feynman gate

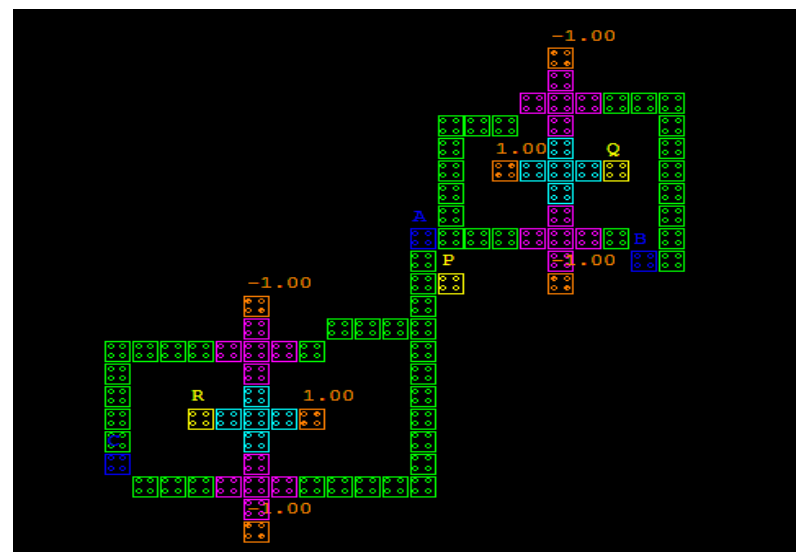

Fig 18: Simulated design of Double Feynman gate

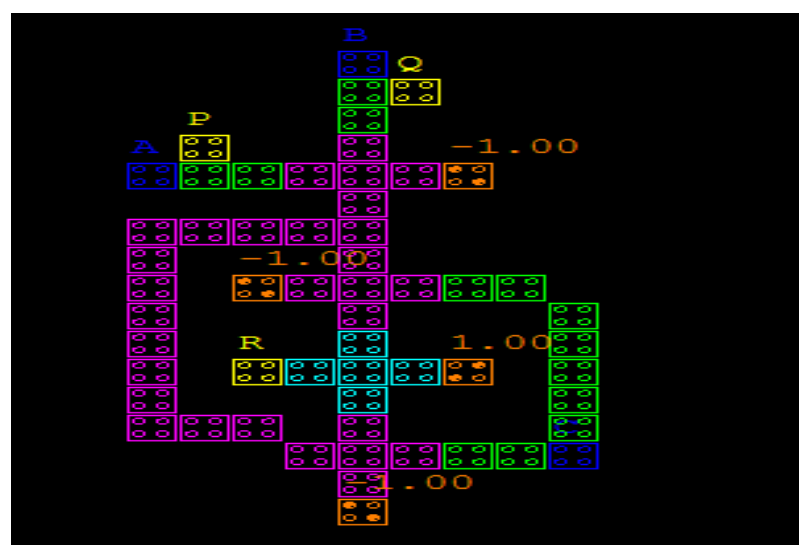

Fig 19: Simulated design of Toffoli gate

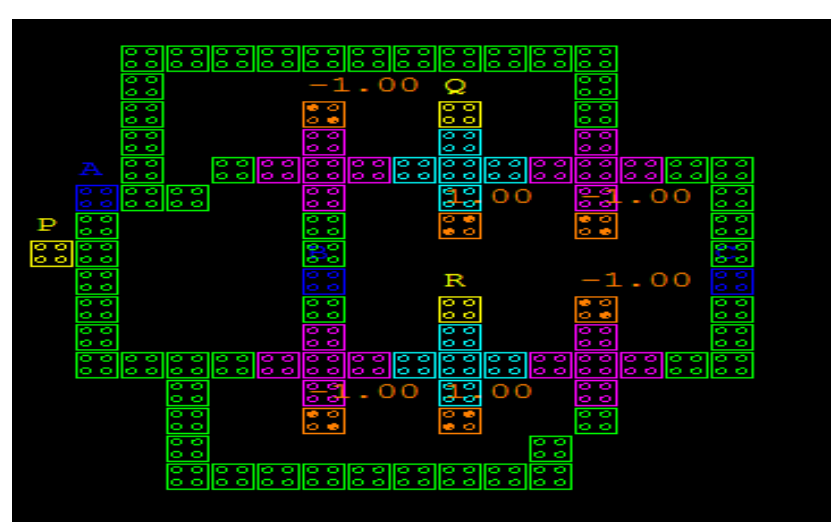

Fig 20: Simulated design of Fredkin gate

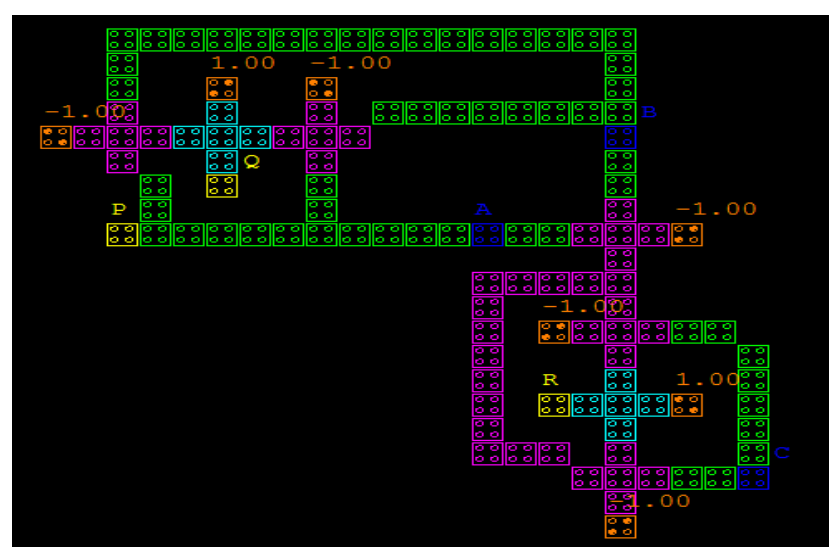

Fig 21: Simulated design of Peres gate

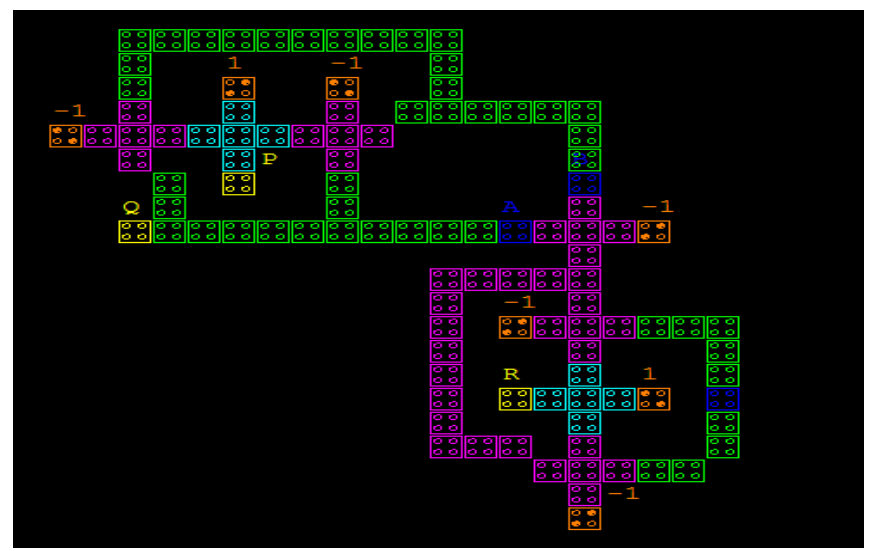

Fig 22: Simulated design of $R$ gate

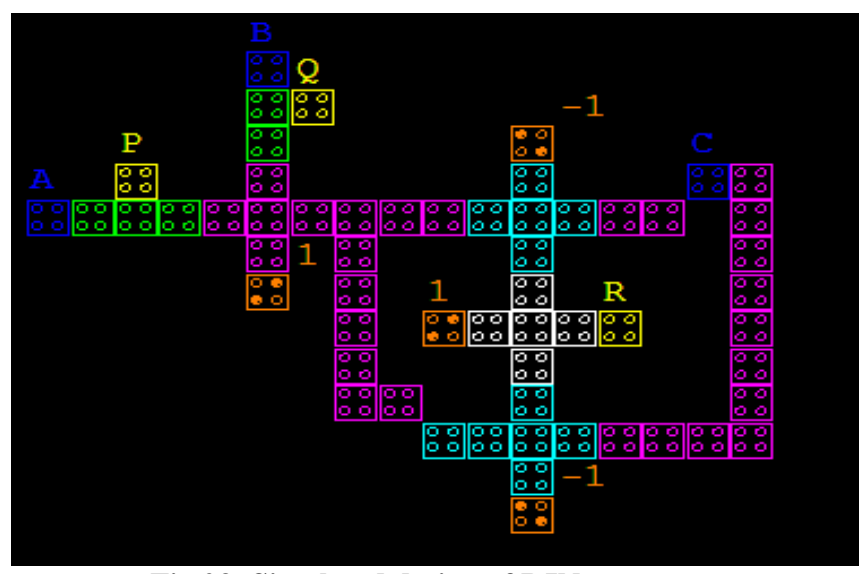

Fig 23: Simulated design of BJN gate 


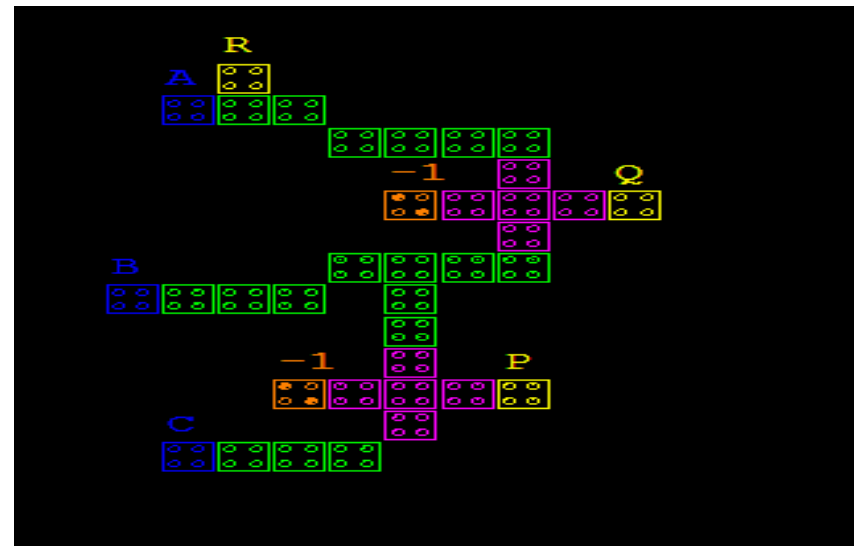

Fig 24: Simulated design of MCL gate

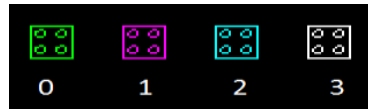

Clock zones

\section{SIMULATION AND RESULTS}

All the design of reversible logic gates were functionally simulated using the QCA Designer 2.0.3. In the bistable approximation, we use the successive criterions that are default criterions in QCADesigner. Cell size $=18 \mathrm{~nm}$, number of samples $=12800$, convergence tolerance $=0.001000$, radius of effect $=65.000000 \mathrm{~nm}$, relative permittivity $=12.900000$, clock high $=9.800000 \mathrm{e}-022 \mathrm{~J}$, clock low $=3.800000 \mathrm{e}-023 \mathrm{~J}$, clock shift $=0$, clock amplitude factor $=2.000000$, layer separation $=11.500000$ and maximum iterations per sample $=100$. The simulation outcomes of reversible gates are shown below:

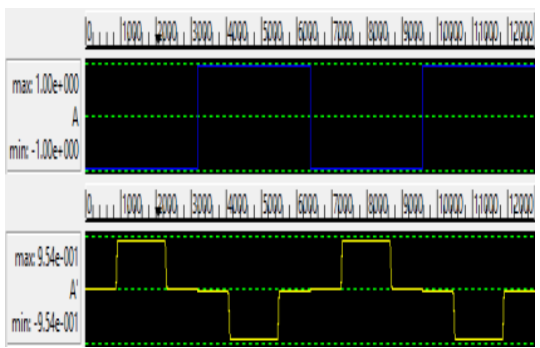

Fig 25: Simulated waveforms for NOT gate

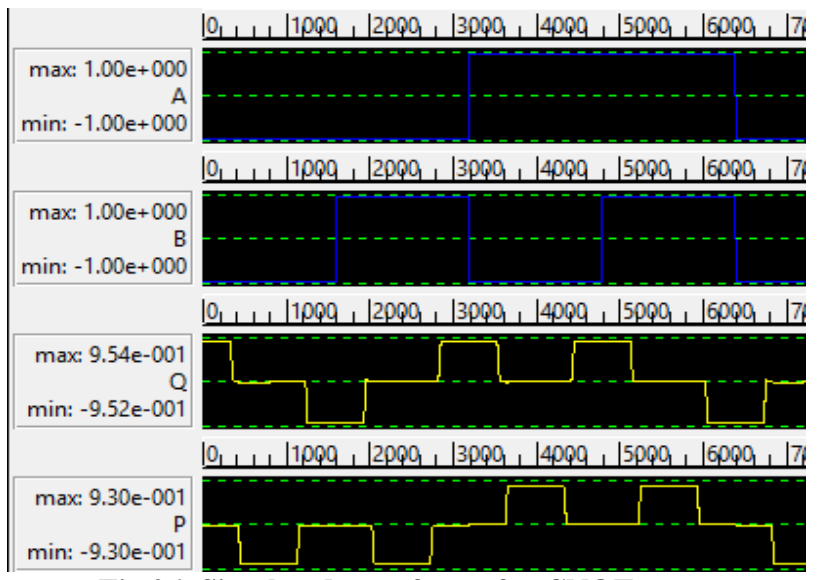

Fig 26: Simulated waveforms for CNOT gate

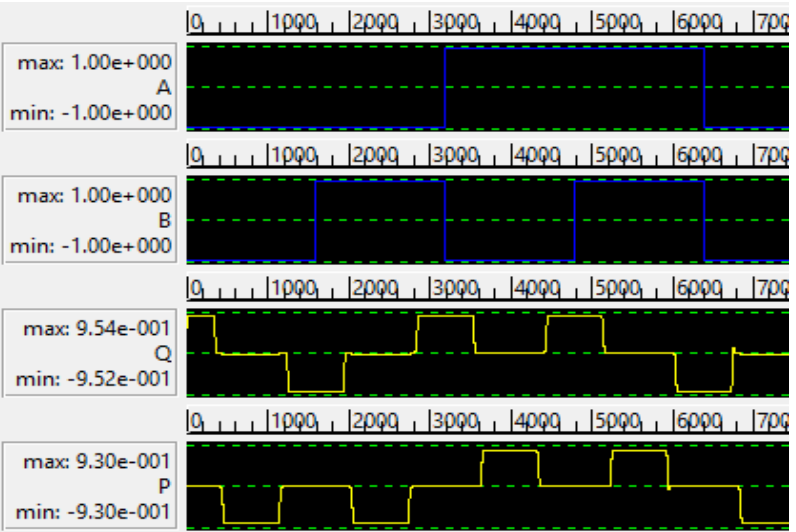

Fig 27: Simulated waveforms for Feynman gate

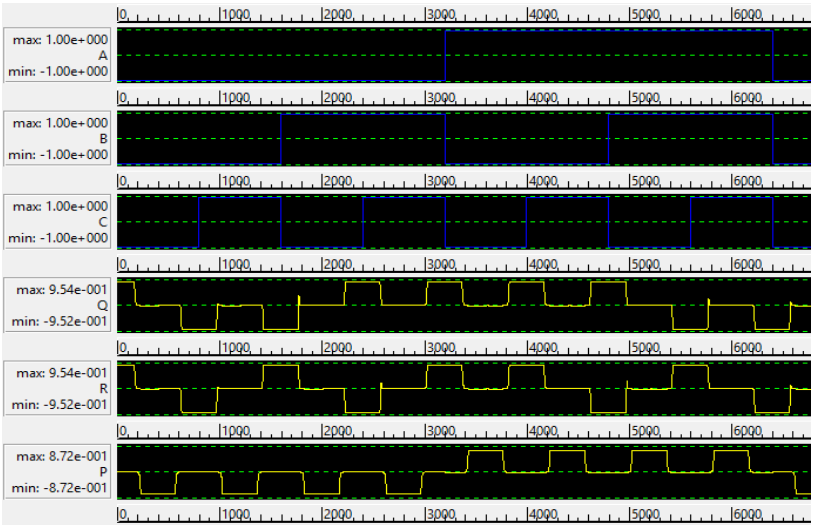

Fig 28: Simulated waveforms for Double Feynman gate

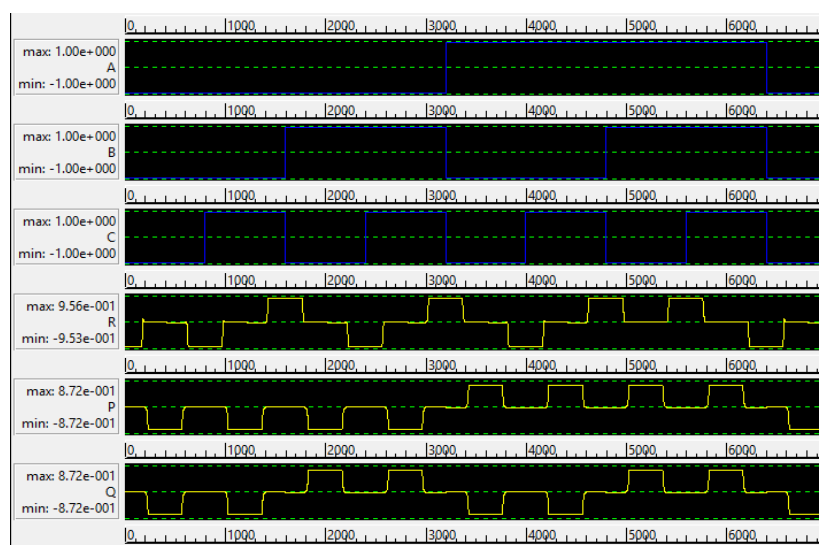

Fig 29: Simulated waveforms for Toffoli gate

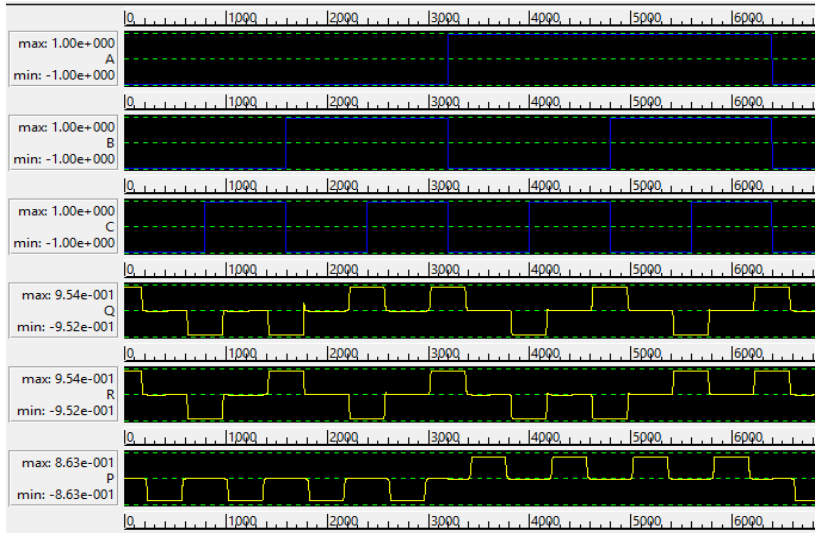

Fig 30: Simulated waveforms for Fredkin gate 


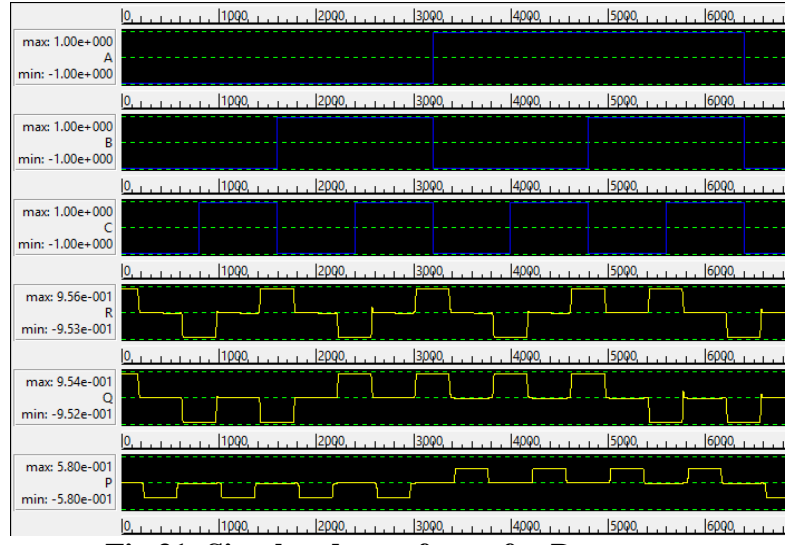

Fig 31: Simulated waveforms for Peres gate

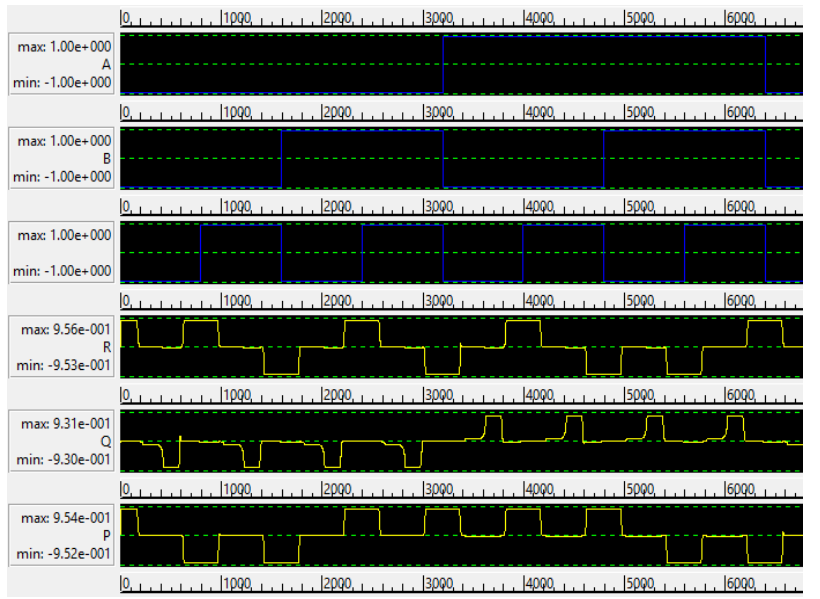

Fig 32: Simulated waveforms for $\mathbf{R}$ gate

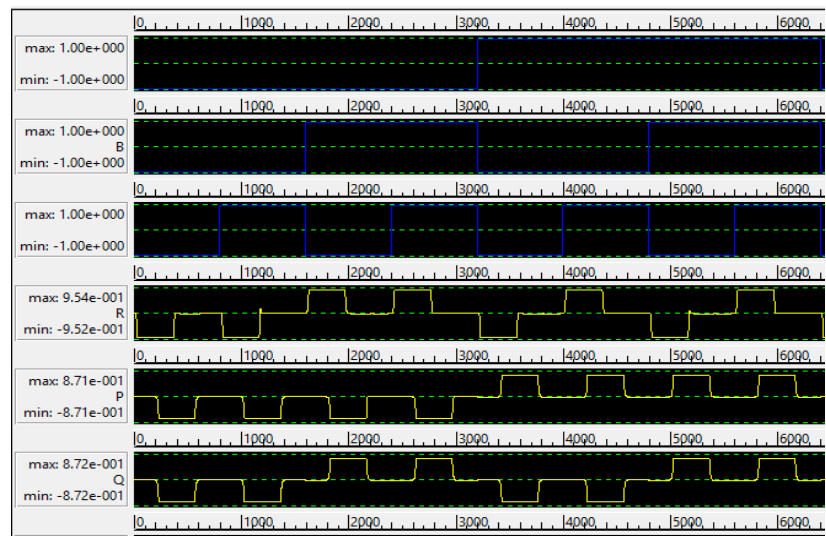

Fig 33: Simulated waveforms for BJN gate

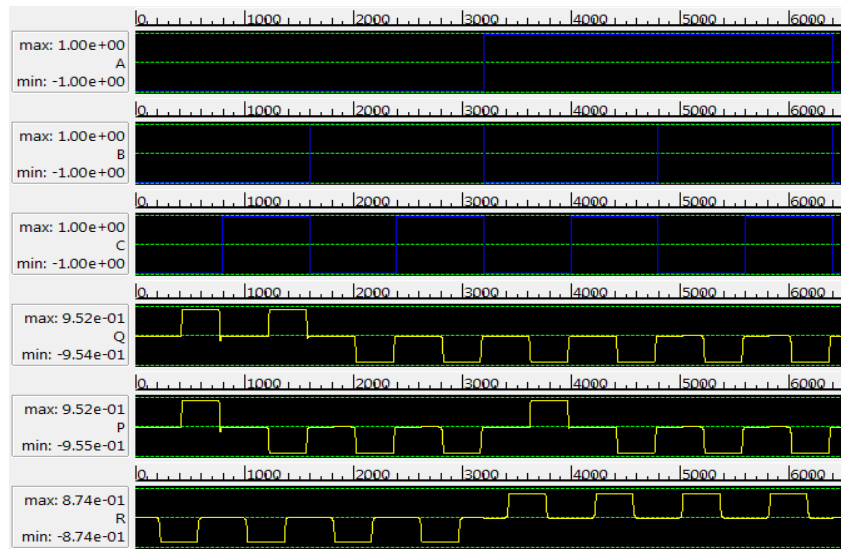

Fig 34: Simulated waveforms for MCL gate
Table 21. Performance Comparison of QCA Implemented Reversible Gates

\begin{tabular}{|c|c|c|c|c|}
\hline \multirow{2}{*}{$\begin{array}{c}\text { Reversible } \\
\text { logic gates }\end{array}$} & \multicolumn{4}{|c|}{ Parameter } \\
\cline { 2 - 5 } & $\begin{array}{c}\text { Number } \\
\text { of cells }\end{array}$ & $\begin{array}{c}\text { Number } \\
\text { of Clock }\end{array}$ & $\begin{array}{c}\text { Clock } \\
\text { delay }\end{array}$ & $\begin{array}{c}\text { Area } \\
\left(\boldsymbol{\mu m ^ { 2 } )}\right.\end{array}$ \\
\hline NOT & 4 & 1 & 0.25 & $0.00 \mu \mathrm{m}^{2}$ \\
\hline CNOT & 49 & 3 & 0.75 & $0.06 \mu \mathrm{m}^{2}$ \\
\hline Feynman & 53 & 3 & 0.75 & $0.07 \mu \mathrm{m}^{2}$ \\
\hline $\begin{array}{c}\text { Double } \\
\text { Feynman }\end{array}$ & 93 & 3 & 0.75 & $0.19 \mu \mathrm{m}^{2}$ \\
\hline Toffoli & 57 & 3 & 0.75 & $0.06 \mu \mathrm{m}^{2}$ \\
\hline Fredkin & 97 & 3 & 0.75 & $0.10 \mu \mathrm{m}^{2}$ \\
\hline Peres & 117 & 3 & 0.75 & $0.18 \mu \mathrm{m}^{2}$ \\
\hline R & 107 & 3 & 0.75 & $0.17 \mu \mathrm{m}^{2}$ \\
\hline BJN & 58 & 4 & 1 & $0.09 \mu \mathrm{m}^{2}$ \\
\hline MCL & 36 & 2 & 0.50 & $0.05 \mu \mathrm{m}^{2}$ \\
\hline
\end{tabular}

\section{CONCLUSION}

This paper presents the conventional reversible gates and their implementation using quantum dot cellular automata. The simulation outcomes show that the proposed circuits execute well. During the simulation a consideration is made to reduce the cell number and the area. The paper can further be extended towards the design of combinational circuits and digital design development using reversible logic circuits which are convenient in quantum computing, nanotechnology, digital signal processing (DSP). The proposed design could be a promising step towards the objective of low power architecture in nanotechnology.

\section{REFERENCES}

[1] Lent, C. S., Tougaw, P. D., \& Porod, W. (1994, November). Quantum cellular automata: the physics of computing with arrays of quantum dot molecules. In Physics and Computation, 1994. PhysComp'94, Proceedings., Workshop on (pp. 5-13). IEEE.

[2] Smith, C. G. (1999). Computation without current. Science, 284(5412), 274

[3] Cho, H., \& Swartzlander Jr, E. E. (2009). Adder and multiplier design in quantum-dot cellular automata. Computers, IEEE Transactions on, 58(6), 721-727.

[4] Frost, S. E., Rodrigues, A. F., Janiszewski, A. W., Rausch, R. T., \& Kogge, P. M. (2002, February). Memory in motion: A study of storage structures in QCA. In First Workshop on Non-Silicon Computing (Vol. 2).

[5] Niemier, M. T., \& Kogge, P. M. (1999). Logic in wire: using quantum dots to implement a microprocessor. In Electronics, Circuits and Systems, 1999. Proceedings of ICECS'99. The 6th IEEE International Conference on (Vol. 3, pp. 1211-1215). IEEE.

[6] Amlani, I., Orlov, A. O., Toth, G., Bernstein, G. H., Lent, C. S., \& Snider, G. L. (1999). Digital logic gate using quantum-dot cellular automata. Science, 284(5412), 289291.

[7] Landauer, R. (1961). Irreversibility and heat generation in the computing process. IBM journal of research and development, 5(3), 183-191. 
[8] Bennett, C. H. (1973). Logical reversibility of computation. IBM journal of Research and Development, 17(6), 525-532.

[9] Merkle, R. C. (1993). Two types of mechanical reversible logic. Nanotechnology, 4(2), 114.

[10] Nielsen, M. A., \& Chuang, I. L. (2010). Quantum computation and quantum information. Cambridge university press.

[11] Knill, E., Laflamme, R., \& Milburn, G. J. (2001). A scheme for efficient quantum computation with linear optics. Nature, 409(6816), 46-52.

[12] Vedral, V., Barenco, A., \& Ekert, A. (1996). Quantum networks for elementary arithmetic operations. Physical Review A, 54(1), 147.

[13] Raghu kanth, B., Murali Krishna, B., Sridhar, M., and Santhi Swaroop, V.G. (2012). A Distinguish Between Reversible and Conventional Logic Gates. International Journal of Engineering Research and Applications, 2(2), 148-151.

[14] Saeedi, M., \& Markov, I. L. (2013). Synthesis and optimization of reversible circuits-a survey. ACM Computing Surveys (CSUR), 45(2), 21.

[15] Thapliyal, H., \& Ranganathan, N. (2010). Design of reversible sequential circuits optimizing quantum cost, delay, and garbage outputs. ACM Journal on Emerging Technologies in Computing Systems (JETC), 6(4), 14.

[16] Bhagyalakshmi, H. R., \& Venkatesha, M. K. (2010). Optimized reversible BCD adder using new reversible logic gates. arXiv preprint arXiv:1002.3994.

[17] Haghparast, M., \& Navi, K. (2008). A novel reversible BCD adder for nanotechnology based systems. American Journal of Applied Sciences, 5(3), 282-288.

[18] Sayem, A. S. M., \& Ueda, M. (2010). Optimization of reversible sequential circuits. arXiv preprint arXiv:1006.4570.

[19] Tougaw, P. D., \& Lent, C. S. (1994). Logical devices implemented using quantum cellular automata. Journal of Applied physics, 75(3), 1818-1825.

[20] Lent, C. S., \& Tougaw, P. D. (1997). A device architecture for computing with quantum dots. Proceedings of the IEEE, 85(4), 541-557.

[21] Benjamin, S. C., \& Johnson, N. F. (1997). A possible nanometer-scale computing device based on an adding cellular automaton. Applied Physics Letters, 70(17), 2321-2323.

[22] Roohi, A., Khademolhosseini, H., Sayedsalehi, S., \& Navi, K. (2011). A novel architecture for quantum-dot cellular automata multiplexer. International Journal of Computer Science Issues, 8(1).

[23] Imre, A., Csaba, G., Ji, L., Orlov, A., Bernstein, G. H., \& Porod, W. (2006). Majority logic gate for magnetic quantum-dot cellular automata. Science, 311(5758), 205-
208

[24] Morita, K. (2008). Reversible computing and cellular automata-A survey. Theoretical Computer Science, 395(1), 101-131.

[25] Bahar, A.N., Waheed, S., and Habib, M.A. 2014. A novel presentation of reversible logic gate in Quantumdot Cellular Automata (QCA). 1st International Conference on Electrical Engineering and Information Communication Technology (ICEEICT), IEEE, 1-6.

[26] Parhami, B. (2006, October). Fault-tolerant reversible circuits. In Signals, Systems and Computers, 2006. ACSSC'06. Fortieth Asilomar Conference on (pp. 17261729). IEEE.

[27] Bahar, A.N., Waheed, S., Uddin, M, A., and Habib, M.A (2013). Double Feynman Gate (F2G) in Quantum-dot Cellular Automata (QCA). International Journal of Computer Science Engineering, 2(6), 351-355.

[28] Toffoli, T. (1980). Reversible computing Tech memo MIT. M IT/LCS/TM-151, MIT Lab for Comp. Sci.

[29] Bahar, A. N., Habib, M., \& Biswas, N. K. (2013). A novel presentation of toffoli gate in quantum-dot cellular automata (QCA). Int J Comput Appl, 82(10), 1-4.

[30] Toffoli, T. (1980). Reversible computing (pp. 632-644) Springer Berlin Heidelberg.

[31] Bahar, A. N., Waheed, S., \& Habib, M. A. (2015). An Efficient Layout Design of Fredkin Gate in Quantum-dot Cellular Automata (QCA). Düzce Üniversitesi Bilim ve Teknoloji Dergisi, 3(1), 219-225.

[32] Peres, A. (1985). Reversible logic and quantum computers. Physical review A, 32(6), 3266.

[33] Sarker, A., Bahar, A.N., Biswas, P.K., and Morshed, M. (2014). A Novel Presentation of Peres Gate (PG) in Quantum-Dot Cellular Automata (QCA), European Scientific Journal. 10 (21), 101-106.

[34] Vasudevan, D. P., Lala, P. K., Di, J., \& Parkerson, J. P. (2006). Reversible-logic design with online testability. Instrumentation and Measurement, IEEE Transactions on, 55(2), 406-414

[35] Bahar, A. N., Waheed, S., \& Hossain, N. (2015). A new approach of presenting reversible logic gate in nanoscale. SpringerPlus, 4(1), 153.

[36] Islam, S., Farzana, S., \& Ali Newaz Bahar, S. (2014). Area Efficient layout design of Multiply Complements Logic (MCL) Gate using QCA Technology. Global Journal of Researches in Engineering, 14(4), 7-10.

[37] Walus, K., Dysart, T. J., Jullien, G., \& Budiman, R. A (2004). QCADesigner: A rapid design and simulation tool for quantum-dot cellular automata. Nanotechnology, IEEE Transactions on, 3(1), 26-31.

[38] "QCADesigner "http://www.mina.ubc.ca/qcadesigner (accessed April 2015). 